\title{
DESENVOLVIMENTO E AVALIAÇÃO DA QUALIDADE DE SORVETE DE IOGURTE SIMBIÓTICO, DE LEITE DE BÚFALA ENRIQUECIDO COM POLPA DE AÇAÍ (Euterpe oleracea)
}

\author{
MONTEIRO, Ranna Catarine da Rocha ${ }^{1}$ \\ VELOSO, Carlos Ribeiro ${ }^{1}$ \\ NERES, Lilaine de Sousa ${ }^{2}$ \\ LOURENÇO JÚNIOR, José de Brito ${ }^{3}$ \\ PACHECO, Evelyn Azevedo ${ }^{1}$ \\ ABE SATO, Suenne Taynah ${ }^{4}$ \\ SANTOS, Marcos Antônio Souza dos ${ }^{5}$ \\ NAHUM, Benjamim de Souza ${ }^{6}$ \\ RIBEIRO, Illana de Araujo ${ }^{7}$
}

\begin{abstract}
RESUMO: Os alimentos funcionais, como prebióticos e probióticos, garantem microbiota intestinal equilibrada e saudável e auxiliam na prevenção de doenças crônico-degenerativas, porém, pouco uso se faz desses alimentos devido à dificuldade de desenvolver produtos com características sensoriais atrativas e micro-organismos viáveis durante toda a vida útil do produto. O objetivo deste estudo foi desenvolver um sorvete de iogurte simbiótico enriquecido com polpa de açaí (Euterpe oleracea), elaborado com leite de búfala. Para o preparo do iogurte, o leite foi filtrado, desnatado e pasteurizado, e posteriormente, acrescido de $1 \%$ de prebiótico (inulina), $0,5 \%$ de cultura láctea (probiótico) e $8 \%$ de sacarose. Após, o iogurte foi dividido para obter quatro formulações de sorvete, F1 - com gordura vegetal e corante; F2 - com gordura vegetal sem corante; F3 - com nata e corante e F4 - com nata sem corante, para verificar a gordura mais acessível e o comportamento do consumidor em relação à adição de corante. $\mathrm{Na}$ avaliação das características físico-químicas, a $\mathrm{F} 1$ se destacou significativamente $(\mathrm{p}<0,05)$, em relação à qualidade nutricional. As formulações receberam notas médias acima de 6, para sabor, cor, textura, aroma e aparência global, de acordo com a escala hedônica de nove pontos, entretanto, as formulações com corante, F1 e F3, destacaram-se significativamente quando relacionadas a F2 e F4 (p>0,05). De acordo com a análise microbiológica os derivados estavam adequados para consumo humano. Assim, a elaboração do sorvete de iogurte enriquecido com açaí, é viável pela sua funcionalidade e valor agregado, com uso de nata ou gordura vegetal e adição de corante.
\end{abstract}

Palavras-chave: Alimento funcional. Derivado lácteo. Produto regional.

SUMMARY: Functional foods as prebiotics and probiotics, ensure balanced and healthy intestinal flora and help in preventing chronic degenerative diseases, however, little use is made of these foods because of the difficulty of developing products with attractive sensory characteristics and viable micro-organisms throughout the useful life of the product. The objective of this study was to develop a symbiotic yogurt ice cream enriched with açaí (Euterpe oleracea) prepared from buffalo milk. For the preparation of yoghurt, the milk was filtered, skimmed, pasteurized, and was increased by $1 \%$ prebiotic (inulin), $0.5 \%$ milk culture (probiotic) and $8 \%$ sucrose. After the yogurt was divided to get four ice cream formulations, F1 - with vegetable fat and dye; F2 - with vegetable fat without dye; F3 with cream and dye and F4 - with cream without dye, to verify the most low fat and consumer behavior in relation to the addition of dye. In the evaluation of physico-chemical characteristics, F1 stood out significantly $(p<0.05)$ in relation to nutritional quality. Formulations received above average grades 6 to flavor, color, texture, flavor and

\footnotetext{
${ }^{1}$ Tecnóloga de Alimentos - Universidade do Estado do Pará

${ }^{2}$ Tecnóloga de Alimentos (UEPA). Cursando pós graduação, nível Mestrado, na UFPA

${ }^{3}$ Engenheiro Agrônomo. Doutor em Ciências Biológicas. Professor Adjunto da Universidade do Estado do Pará.

${ }^{4}$ Tecnóloga de Alimentos. Nutricionista. Cursando Mestrado em Ciência e Tecnologia de Alimentos na Universidade Federal do Pará.

${ }^{5}$ Engenheiro Agrônomo; Mestre em Economia; Professor da Universidade Federal Rural da Amazônia (UFRA).

${ }^{6}$ Médico Veterinário. Mestre em Ciência Animal pela Universidade Federal do Pará. Pesquisador B da Embrapa Amazônia Oriental.

${ }^{7}$ Engenheira de Pesca. Técnica do Laboratório de Alimentos da Universidade do Estado do Pará.
} 
overall appearance, according to the nine-point hedonic scale, however, the dye formulations, F1 and F3, stood out as significantly related to F2 and F4 ( $>$ > 0.05). In accordance with the microbiological analysis were derivatives suitable for human consumption. Thus, the development of symbiotic ice cream yogurt flavor acai is viable for its functionality and value, using cream or vegetable fat and adding dye.

Keywords: Functional food. Dairy products. Regional product.

\section{INTRODUÇÃO}

A bubalinocultura de leite constitui atividade que conquista a pecuária brasileira, sendo predominante na Amazônia Oriental, sobretudo na região do Arquipélago do Marajó, em propriedades rurais de base familiar, constituindo fonte de alimentação e renda (TEIXEIRA et al., 2005; LOURENÇO JÚNIOR; GARCIA, 2006).

O leite de búfala possui elevados níveis de proteínas e minerais, em especial cálcio e fósforo, além do destacado aproveitamento industrial, superior em $40 \%$ ao leite bovino. Pode ser comercializado in natura e/ou transformado em derivados, como queijos, iogurtes, doce de leite, agregando valor à matéria prima, além de incrementar a renda familiar (VIEIRA et al., 2005; ZACARCHENCO et al., 2009).

Atualmente, saúde e bem-estar são focos de pesquisas da indústria de alimentos, os quais devem contribuir com o bom funcionamento do organismo humano. Os alimentos funcionais, como prebióticos e probióticos, garantem microbiota intestinal equilibrada e saudável que auxiliam na prevenção de doenças crônico-degenerativas (SANTOS; CANÇADO, 2009). Entretanto, faz-se pouco uso desses alimentos, uma vez que pesquisas esbarram na dificuldade de desenvolver produtos com características sensoriais atrativas e micro-organismos viáveis, durante toda a vida útil do produto. O sorvete de iogurte é diferente dos demais sorvetes, pois há fermentação prévia, e parte dos açúcares é transformada em ácido láctico, pela ação de bactérias (ALAMPRESE et al., 2005; ALVES et al., 2009).

$\mathrm{Na}$ Amazônia, o açaí (Euterpe oleracea Mart.) é muito consumido pela população regional devido ao seu sabor exótico e fonte de importantes nutrientes, e só no estado do Pará estão $95 \%$ da sua produção (MENEZES et al., 2008; RUFINO et al., 2011). Esse produto tem sido comercializado de diversas formas, tais como, polpa pasteurizada, açaí com xarope de guaraná, açaí em pó, doce de leite com açaí e geleia. O crescente interesse e investimentos em pesquisas sobre o aproveitamento tecnológico desse fruto pode ser atribuído às suas propriedades nutricionais, pois é rico em proteínas, fibras, lipídios, dos quais $60 \%$ monoinsaturado e $13 \%$ poli-insaturado, vitamina E e minerais. Além disso, os benefícios do açaí no perfil lipídico foram identificados na atenuação de doenças cardiovasculares em coelhos hipercolesterolêmicos (ROGEZ, 2000; OLIVEIRA et al., 2007; FEIO et al., 2012).

Assim, esta pesquisa visou elaborar um sorvete de iogurte, à base de leite búfala com propriedade simbiótica e saborizado com açaí, a fim de avaliar suas características físico-química, microbiológica e sensorial.

\section{MATERIAL E MÉTODO}

\section{Elaboração do sorvete de iogurte de búfala enriquecido com açaí}

O leite de búfala foi obtido na Unidade de Pesquisa Animal "Dr. Felisberto Camargo" (01²6'S e $48^{\circ} 24^{\prime}$ W), da Embrapa Amazônia Oriental. A polpa de açaí in natura e demais ingredientes foram obtidos no mercado local. A elaboração do derivado e as análises foram feitas no Laboratório de Tecnologia de 
Alimentos do Centro de Ciências Naturais e Tecnologia - CCNT, da Universidade do Estado do Pará UEPA, em Belém, Pará.

Após recepção, o leite foi filtrado, desnatado e pasteurizado a $90^{\circ} \mathrm{C}$ por cinco minutos (GONÇALVES; EBERLE, 2008). No preparo do iogurte foram utilizadas duas culturas lácteas concentradas, uma starter (Streptococcus thermophilus e Lactobacillus bulgaricus) e outra probiótica (Bifidobacterium e Lactobacillus acidophilus). Um grama de cada cultura (1,0 x 10 ${ }^{6}$ UFC g-1 de cada micro-organismo) foi inoculado em $100 \mathrm{ml}$ de leite bubalino, denominada cultura-mãe.

O leite foi foi acrescido de $8 \%$ de sacarose (m/v), $1 \%$ de inulina (prebiótico, $\mathrm{m} / \mathrm{v}$ ) e $0,5 \%$ de cultura-mãe $(\mathrm{m} / \mathrm{v})$, incubada a $42^{\circ} \mathrm{C}$, até $\mathrm{pH} 4,8$, durante quatro horas. Após a fermentação, dividiu-se o iogurte para elaborar quatro formulações de sorvete (Tabela 1). O iogurte foi batido com os ingredientes de cada formulação, a fim de obter um produto homogêneo com consistência e aeração típicas. O derivado foi acondicionado em embalagens plásticas $(100 \mathrm{~mL})$ e mantido em freezer $\left( \pm 2^{\circ} \mathrm{C}\right)$, até as análises.

Tabela 1. Percentual de constituintes adicionados ao iogurte em cada formulação de sorvete simbiótico de leite de búfala enriquecido com polpa de açaí.

\begin{tabular}{lcccc}
\hline Componente & F1 (\%) & F2 (\%) & F3 (\%) & F4 (\%) \\
\hline Nata $(\mathrm{m} / \mathrm{m})$ & 10 & 10 & - & - \\
Gordura vegetal $(\mathrm{m} / \mathrm{m})$ & - & - & 10 & 10 \\
Xarope de glucose $(\mathrm{m} / \mathrm{m})$ & 7 & 7 & 7 & 7 \\
Emulsificante/Estabilizante $(\mathrm{m} / \mathrm{m})$ & 1 & 1 & 1 & 1 \\
Liga neutra $(\mathrm{v} / \mathrm{m})$ & 1 & 1 & 1 & 1 \\
Polpa de açaí $(\mathrm{v} / \mathrm{m})$ & 4 & 4 & 4 & 4 \\
Corante $(\mathrm{v} / \mathrm{m})$ & 0,3 & - & 0,3 & -
\end{tabular}

Notas: F1: Sorvete de iogurte com nata e corante; F2: Sorvete de iogurte sem corante e com nata; F3: Sorvete de iogurte com corante e com gordura vegetal; F4: Sorvete de iogurte sem corante e com gordura vegetal.

Durante o processo de bateção houve incorporação de ar ao sorvete de iogurte, que resulta em aumento da mistura inicial, isto é, overrun, que foi calculado pela fórmula descrita por Mosquim (1999).

\section{Análises físico-químicas}

A caracterização físico-química foi realizada em triplicata: $\mathrm{pH}$, medido com potenciômetro portátil; proteína bruta, método micro Kjedahl; lipídios totais, pelo método Gerber; umidade; resíduo mineral fixo (AOAC, 1997); determinação da acidez em graus Dornic; determinação do extrato seco total; e determinação do extrato seco desengordurado foi realizada de acordo com o Instituto Adolfo Lutz (2005).

\section{Análises microbiológicas}

As análises microbiológicas do leite e derivado seguiram a metodologia de Silva et al. (2001) e os resultados foram avaliados conforme a legislação vigente (BRASIL, 2001). As análises microbiológicas foram coliformes totais a $45^{\circ} \mathrm{C}$, Staphylococcus coagulase positiva e Salmonella sp. 


\section{Análise sensorial}

Foi realizado teste sensorial de aceitação com 50 provadores, de ambos os sexos e não treinados. Foram fornecidos $50 \mathrm{~g}$ de cada formulação do sorvete de iogurte simbiótico, identificadas com números aleatórios de três dígitos, além da ficha de avaliação sensorial para avaliação dos atributos sensoriais, cor, aroma, sabor, textura e aceitação global, de acordo com escala hedônica de 9 pontos (DUTCOSKY, 2007). Foi oferecido aos provadores água mineral para limpeza do palato entre as amostras.

\section{Análise estatística}

A análise estatística considerou os resultados obtidos nos quatro tratamentos e três repetições utilizando-se o programa BioEstat 5.0 (AYRES et al., 2007). Os dados foram analisados de forma descritiva quantitativa e submetidos à análise de variância (ANOVA). As variáveis de resposta foram comparadas entre si, utilizando-se teste Tukey, em nível de significância de 5\%.

\section{RESULTADO E DISCUSSÃO}

\section{Análises físico-químicas}

A legislação vigente não contempla padrões de $\mathrm{pH}$ e acidez para gelados comestíveis, e os resultados encontrados não diferem entre as amostras $(\mathrm{p}>0,005)$. $O$ teor de umidade diferiu entre as amostras F1 e F3 em relação a F2 e F4. O teor proteico das formulações superou o mínimo recomendado $(2,5 \%)$ pela legislação vigente (BRASIL, 2005). Os teores de lipídios variaram entre 5,8 e 6,5\%, próximos aos recomendados para sorvete de qualidade, em torno de 6\%. Destaca-se que esse componente pode afetar a qualidade do sorvete de iogurte elaborado neste trabalho, pois há relatos de que teor de lipídios de até $5 \%$ produz maior aceitabilidade do derivado, entretanto, no Egito, são recomendados valores superiores, de 10\% (TAMIME; ROBINSON, 2007).

Gonçalves e Eberle (2008), que também encontraram teores de umidade $(73,24 \%)$ e cinzas $(0,79 \%)$ próximos aos do presente trabalho (Tabela 2).

Tabela 2. Caracterização físico-química de sorvete de iogurte simbiótico de leite de búfala enriquecido com polpa de açaí.

\begin{tabular}{lcccc}
\hline \multirow{2}{*}{ Parâmetro } & \multicolumn{4}{c}{ Média (\%) \pm Desvio-padrão } \\
\cline { 2 - 5 } & F1 & F2 & F3 & F4 \\
\hline pH & $4,81^{\mathrm{a}} \pm 0,01$ & $4,71^{\mathrm{a}} \pm 0,02$ & $4,55^{\mathrm{a}} \pm 0,05$ & $4,56^{\mathrm{a}} \pm 0,00$ \\
Umidade & $72,65^{\mathrm{a}} \pm 0,26$ & $74,46^{\mathrm{b}} \pm 0,08$ & $72,66^{\mathrm{a}} \pm 0,23$ & $73,24^{\mathrm{b}} \pm 0,05$ \\
Proteína & $18,25^{\mathrm{a}} \pm 0,01$ & $16,70^{\mathrm{b}} \pm 0,38$ & $18,51^{\mathrm{a}} \pm 0,90$ & $13,43^{\mathrm{c}} \pm 0,89$ \\
Extrato seco total & $27,36^{\mathrm{a}} \pm 0,26$ & $25,54^{\mathrm{b}} \pm 0,08$ & $27,34^{\mathrm{a}} \pm 0,23$ & $26,76^{\mathrm{c}} \pm 0,05$ \\
Extrato seco desengordurado & $21,54^{\mathrm{a}} \pm 0,28$ & $19,39^{\mathrm{b}} \pm 0,04$ & $20,82^{\mathrm{c}} \pm 0,02$ & $20,71^{\mathrm{c}} \pm 0,04$ \\
Lipídios & $5,82^{\mathrm{a}} \pm 0,01$ & $6,15^{\mathrm{b}} \pm 0,32$ & $6,52^{\mathrm{b}} \pm 0,12$ & $6,05^{\mathrm{c}} \pm 0,52$ \\
Cinzas & $0,83^{\mathrm{a}} \pm 0,11$ & $0,70^{\mathrm{b}} \pm 0,002$ & $0,87^{\mathrm{a}} \pm 0,07$ & $0,78^{\mathrm{a}} \pm 0,01$ \\
\hline
\end{tabular}

Notas: ${ }^{\text {abc }}$ Médias seguidas por letras diferentes na linha diferem estatisticamente $(\mathrm{P}<0,05)$. F1: Sorvete de iogurte com corante e nata; F2: Sorvete de iogurte sem corante e com nata; F3: Sorvete de iogurte com corante e gordura vegetal; F4: Sorvete de iogurte sem corante e com gordura vegetal.

O teor de extrato seco total da formulação F1 e F3 estão próximos ao recomendado por Vicente e Castillo (1995), que citam valor ideal de 30\% para manter boa textura do sorvete de iogurte. Gonçalves e 
Eberle (2008) indicam que sorvete de iogurte possui de 7 a $12 \%$ de extrato seco desengordurado, assim, os valores encontrados no presente trabalho foram elevados. Devido ao alto teor lipídico do leite bubalino, embora tenha sido semidesnatado, além da adição de ingredientes gordos, tais como nata ou gordura vegetal.

\section{Análises microbiológicas}

Os resultados das análises microbiológicas do leite pasteurizado e do sorvete de iogurte simbiótico atenderam a legislação vigente (BRASIL, 2001), o que indica ausência de riscos para consumo humano (Tabela 3). Esse fato deve-se ao tratamento térmico eficiente empregado no leite e polpa de açaí, o que garantiu as características sensoriais adequadas, maior aceitabilidade, vida útil prolongada e maior rendimento industrial (TEIXEIRA et al., 2005), bem como obtenção de ingredientes de qualidade e manutenção de boas práticas de fabricação.

Tabela 3. Resultados das análises microbiológicas do leite de búfala semidesnatado e do sorvete de iogurte simbiótico de leite de búfala enriquecido com polpa de açaí.

\begin{tabular}{lcccccccc}
\hline \multirow{2}{*}{ Análise } & \multicolumn{2}{c}{ Leite de búfala } & \multicolumn{5}{c}{ Sorvete de iogurte } \\
\cline { 2 - 9 } & Legislação & Pasteurizado & Legislação & F1 & F2 & F3 & F4 \\
\hline $\begin{array}{l}\text { Coliformes totais } \\
\left(45^{\circ} \mathrm{C} \text { NMP/g) }\right.\end{array}$ & $4 \times 10$ & $<3$ & $5 \times 10$ & $<3$ & $<3$ & $<3$ & $<3$ \\
$\begin{array}{l}\text { Staphylococcus } \\
\text { positiva/g (UFC/g) } \\
\text { Salmonella sp./25g }\end{array}$ & coag. & - & - & $5 \times 10^{2}$ & ND & ND & ND & ND \\
\hline
\end{tabular}

Notas: F1: Sorvete de iogurte com corante e com nata; F2: Sorvete de iogurte sem corante e com nata; F3: Sorvete de iogurte com corante e com gordura vegetal; F4: Sorvete de iogurte sem corante e com gordura vegetal. ND: Nada detectado.

\section{Análise sensorial}

$\mathrm{Na}$ avaliação sensorial das formulações de sorvete de iogurte, todas as amostras obtiveram aceitação satisfatória, uma vez que a maioria dos atributos sensoriais avaliados (sabor, cor, textura, aroma e aparência global) receberam notas médias superiores a 6, "gostei ligeiramente" (Tabela 4). Foram observadas diferenças significativas nos atributos Cor e Aroma, entre as formulações com e sem adição de corante, cujas notas foram superiores para aquelas com corante devido ao visual mais atrativo.

Tabela 4. Teste de aceitação de sorvete de iogurte simbiótico de leite de búfala enriquecido com polpa de açaí.

\begin{tabular}{lcccc}
\hline \multirow{2}{*}{ Atributo } & \multicolumn{4}{c}{ Sorvete de iogurte } \\
\cline { 2 - 5 } & F1 & F2 & F3 & F4 \\
\hline Cor & $8,22^{\mathrm{a}}$ & $5,50^{\mathrm{b}}$ & $8,18^{\mathrm{a}}$ & $5,32^{\mathrm{b}}$ \\
Aroma & $6,30^{\mathrm{a}}$ & $5,82^{\mathrm{b}}$ & $6,18^{\mathrm{a}}$ & $5,88^{\mathrm{b}}$ \\
Textura & $7,42^{\mathrm{a}}$ & $6,00^{\mathrm{b}}$ & $6,16^{\mathrm{b}}$ & $6,76^{\mathrm{a}}$ \\
Sabor & $6,28^{\mathrm{a}}$ & $6,26^{\mathrm{a}}$ & $6,12^{\mathrm{a}}$ & $6,84^{\mathrm{a}}$ \\
Aparência global & $7,38^{\mathrm{a}}$ & $6,40^{\mathrm{a}}$ & $7,24^{\mathrm{a}}$ & $7,12^{\mathrm{a}}$ \\
\hline
\end{tabular}

Notas: ${ }^{\text {ab }}$ Médias seguidas por letras diferentes na linha diferem estatisticamente em 5\% de probabilidade. F1: com gordura vegetal e corante; F2: com gordura vegetal sem corante; F3: com nata e corante e F4: com nata sem corante. 
Quanto à Textura, a diferença observada deve-se à utilização de nata ou gordura vegetal. A gordura promove textura mais macia no sorvete, e iogurtes semidesnatados tendem a apresentar textura mais frágil e quebradiça (THOMOPOULOS, 1993). Paula et al. (2010) afirmam que a adição de gordura vegetal hidrogenada melhora a textura do sorvete.

Com relação ao sabor não foram observadas diferenças significativas $(\mathrm{p}>0,05)$ entre as formulações, mesmo com utilização de dois tipos de gordura (vegetal e nata). Finalmente, para o atributo aparência global, que reflete a somatória dos demais atributos, não foram encontradas diferenças estatísticas entre as formulações.

A quantidade de ar incorporada (overrun), em cada formulação de sorvete de iogurte

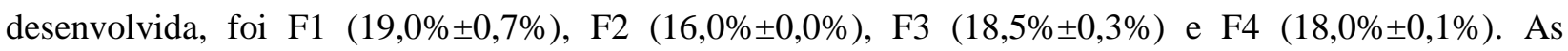
formulações F1, F3 e F4 estiveram próximas da percentagem de overrun citada por Sofjan e Hartel (2004) que estabelece valor mínimo de $20 \%$ para sorvetes, enquanto a F2 apresentou percentagem de overrun muito baixa, o que indica um produto de alta densidade.

\section{CONCLUSÃO}

O sorvete de iogurte simbiótico de leite de búfala enriquecido com polpa de açaí é passível de ser elaborado com gordura vegetal ou nata e corante, pois além de atender aos padrões microbiológicos estabelecidos pela legislação vigente, juntamente com o açaí, resultou em derivado com características sensoriais satisfatórias e apresentou nas formulações manipuladas qualidade nutricional destacada e adaptação para os simbióticos utilizados. Portanto, a combinação de alimento funcional e matérias-primas regionais permite a obtenção de derivado de alto valor agregado, bem como disponibiliza inovação a cadeia produtiva de leite e de frutas na Amazônia.

\section{REFERÊNCIAS}

ALAMPRESE, C. et al. Effects of Lactobacillus rhamnosus GC addition in ice cream. International Journal of Dairy Technology, v.58, n.4, p.200-206, 2005. Disponível em: http://www3.interscience.wiley.com/cgi-bin/fulltext/118668043/PDFSTART

ALVES, L. et al. Aceitação sensorial e caracterização de frozen yogurt de leite de cabra com adição de cultura probiótica e prebiótico. Ciência Rural, v.33, n.2, p.675-686, 2009. Disponível em: http://www.scielo.br/pdf/cr/2009nahead/a361cr1087.pdf

AOAC (ASSOCIATION OF OFFICIAL ANALYTICAL CHEMISTS). Official Methods of Analysis. 16. ed., Virginia, 1997.

AYRES, M. et al. Bio Estat. 5.0. Aplicações estatísticas nas áreas das ciências médicas. 5.ed. Belém, 2007. 339p.

BRASIL. Ministério da Saúde. Agência Nacional de Vigilância Sanitária. Resolução RDC nº 266, de 22 de setembro de 2005. Regulamento técnico para gelados comestíveis e preparados para gelados

comestíveis. Diário Oficial da República Federativa do Brasil, Poder Executivo, Brasília, DF, 23 set. 2005. 
BRASIL. Ministério da Saúde. Resolução RDC nº 12, de 02 de janeiro de 2001. Aprova o Regulamento Técnico sobre Padrões Microbiológicos para Alimentos. Diário Oficial [da] Republica Federativa do Brasil. Brasília, DF, 10 jan. 2001. Seção 1, nº7-E. p.45-53.

DUTCOSKY, S. D. Análise sensorial de alimentos. $2^{\text {a }}$ ed. Curitiba: Champagnat, 2007.

FEIO, C. M. A. et al. Euterpe Oleracea (Açaí) modifies sterol Metabolism and Attenuates ExperimentallyInduced Atherosclerosis. International Journal Atherosclerosis, v. 19, n.3, p.237-245, 2012. Disponível em: http://www.ncbi.nlm.nih.gov/pubmed/22139433

GONÇALVES, A. A.; EBERLE, I. R. Frozen yogurt com bactérias probióticas. Alimentos Nutritivos, v.19, n.3, p.291-297, 2008. Disponível em: http://200.145.71.150/seer/index.php/alimentos/article/viewFile/633/531?origin=publication_detail

INSTITUTO ADOLFO LUTZ. Métodos físico-químicos para análise de alimentos: normas analíticas do Instituto Adolfo Lutz. 4. ed. Brasília: Anvisa, 2005.

LOURENÇO JÚNIOR, J. B.; GARCIA, A. R. Produção animal no Bioma Amazônico: atualidades e perspectivas. Revista Brasileira de Zootecnia, v. 35, n. 1, p. 63-83, 2006.

MENEZES, E. M. S.; TORRES, A.T.; SABAA SRUR, A. U. Valor nutricional da polpa de açaí (Euterpe oleracea Mart.) liofilizada. Acta Amazônica, v.38, n.2, p. 311-316, 2008. Disponível em: http://www.scielo.br/pdf/aa/v38n2/v38n2a14.pdf

MOSQUIM, M. C. A. Fabricando sorvete com qualidade. São Paulo: Varela, 1999.

OLIVEIRA, M. S. P.; FARIAS NETO, J. T.; PENA, R. S. Açaí: técnicas de cultivo e processamento. Fortaleza: Instituto Frutal, 2007.

PAULA, C. M. et al. Sorvete potencialmente probiótico de leite de cabras, sabor morango, adoçado com mel de abelhas africanizadas. Coletânea BITEC 2008-2010. Brasília: IEL - Instituto Euvaldo Lodi, SEBRAE e CNPq, v.1, n.8, p.89-102, 2010.

ROGEZ, H. Açaí: preparo, composição e melhoramento da conservação. Belém: Editora da Universidade Federal do Pará (EDUPA), 2000.

RUFINO, M. S. M. et al. Açaí (Euterpe oleracea) BRS Pará: A tropical fruit source of antioxidant dietary fiber and high antioxidant capacity oil. Food Research International, v.44, n.7, p.2100-106, 2011. Disponível em: http://www.sciencedirect.com/science/article/pii/S0963996910003194

SANTOS, L. C.; CANÇADO, I. A. C. Probióticos e prebióticos: vale a pena incluí-los em nossa alimentação. SynThesis, v.1, n.1, p.308-17, 2009. Disponível em:

http://www.fapam.edu.br/revista/upload/8092009180630Alimentos funcionais corrigido NUPE 29-0409.pdf

SILVA, N.; JUNQUEIRA, V. C. A.; SILVEIRA, N. F. A. Manual de métodos de análise microbiológica de alimentos. $2^{\text {a }}$ ed. São Paulo: Varela, 2001.

SOFJAN, R. P.; HARTEL, R. W. Effects of overrun on structural and physical characteristics of ice cream. International Dairy Journal, v.14, n.1, p.255-262, 2004. Disponível em: http://www.sciencedirect.com/science/article/pii/S0958694603001973

TAMIME, A. Y.; ROBINSON, R. K. Yoghurt: science and technology. $3^{\mathrm{a}}$ ed. England: Woodhead Publishing Ltd and CRC Press LLC, 2007. 
TEIXEIRA, L. V.; BASTIANETTO, E.; OLIVEIRA, D. A. A. Leite de búfala na indústria de produtos lácteos. Revista Brasileira de Reprodução Animal, v.29, n.2, p.96-100, 2005. Disponível em: http://www.cbra.org.br/pages/publicacoes/rbra/download/RE024.pdf

THOMOPOULOS, C.; TZIA, C.; MILKAS, D. Influence of processing of solids fortified milk on coagulation time and quality properties of yogurt. Milchwissenschaft, v. 48, n.8, p.426-30, 1993.

VICENTE, A. M.; CASTILLO, I. C. Tecnología de la elaboración de los helados. Madrid: MundiPrensa; 1995.

VIEIRA, L. C. et al. Produção de requeijão marajoara de leite de búfala. In: $5^{\circ}$ Congresso Brasileiro de Zootecnia, Campo Grande: ZOOTEC. p. 1-4, 2005. [CD-ROM].

ZACARCHENCO, P. B. et al. Desafios tecnológicos na fabricação de queijos e requeijão cremoso de baixo teor de sódio. Revista Indústria de Laticínios, v.14, n.80, p.82-85, 2009. 